\title{
Joint Design of Control and Power Efficiency in Wireless Networked Control System
}

\author{
Yan Wang and Zhicheng Ji \\ Department of IoT Engineering, Jiangnan University, Wuxi 214122, China \\ Correspondence should be addressed to Yan Wang; violetwang0929@gmail.com
}

Received 29 April 2014; Accepted 10 July 2014; Published 6 August 2014

Academic Editor: Xi-Ming Sun

Copyright ( 2014 Y. Wang and Z. Ji. This is an open access article distributed under the Creative Commons Attribution License, which permits unrestricted use, distribution, and reproduction in any medium, provided the original work is properly cited.

\begin{abstract}
This paper presents a joint design method for wireless networked control system (WNCS) to balance both the demands of network service and the control performance. Since the problems of power consumption, communication reliability, and system stability exist simultaneously and interdependently in WNCS, most of the achieved results in the wireless network and wired networked control system cannot be used directly. To coordinate the three problems, sampling period is found to be the linking bridge. An adaptive sampling power efficiency algorithm is proposed to manage the power consumption such that it can meet the demands of network life span. The sampling period is designed to update periodically on the constraints of network schedulability and system stability. The convergence of the power efficiency algorithm is further proved. The sampling period is no longer a fixed value, however; thus, increasing the difficulty in modeling and controlling such a complicated time-varying system remains. In this work, a switched control system scheme is applied to model such a WNCS, and the effect of network-induced delay is considered. Switched feedback controllers are introduced to stabilize the WNCS, and some considerations on stability condition and the bounds of the update circle for renewing sampling period are discussed. A numerical example shows the effectiveness of the proposed method.
\end{abstract}

\section{Introduction}

Wireless networked control systems are composed of distributed fields and plant devices (sensors, actuators, and controllers) interconnected via a wireless network [1]. The sensors, controllers, and actuators exchange information with one another through the wireless network. Sensors collect the status or outputs of plants at every sampling instant, packet the data with time-stamp, and then send the information to each corresponding controller via the wireless network. Controllers then compute the control variables as soon as they receive the newest data from their plants. After that, the control variables are sent to their corresponding actuators. Plants will not update their status until they receive the newest command from controllers. Wireless capabilities clearly provide opportunities to be more inventive in system organization [2]. The use of wireless network offers several advantages compared with a conventional wired networked control system in terms of cost, maintenance, scalability, and implementation flexibility. However, wireless nodes also have obvious weak points such as reliability and availability, particularly the power consumption. Power efficiency technologies are important research areas in wireless network applications.

Most studies on WNCS analyze the effects of the wireless medium on overall closed-loop control systems in [3-11]. The first critical analysis on the use of wireless control is performed by Kumar in [3]. Kumar explores the impact of different protocol layers, from routing to physical, on control performance. In [4], Liu and Goldsmith analyzed the performance of a control system in terms of variation in data rate, error correcting codes, and different maximum bounds in the number of retransmissions. They also studied the impact of IEEE 802.11b medium access control. In [5], Colandairaj et al. discuss the impact of data flow on the stability of a closed-loop wireless network control system based on IEEE 802.11. Sampling rate adaption is proposed as a codesign solution to enhance control and wireless network performance. Different from our work in this paper, 
the purpose of adapting the sampling rate is to optimize bandwidth utilization not to save power. A robust and dynamic cross-layer communication architecture for wireless networked control system is presented in [6] by Israr et al. The protocol stack for WNCS comprises five layers. Each layer contributes to the overall goal of reliable, power-efficient communication. However, the control performance is not taken into account in their work.

A number of studies related to power efficiency in wireless sensor networks (WSNs) and wireless networks have also been conducted in [12-18]. Current power efficiency research always falls into two categories: one is reducing the power consumption of each single node in the network and the other is balancing the power consumption of all the nodes in network. In [12], Colandairaj et al. present a dynamic power control strategy to minimize the communication power consumption of nodes by varying the transmission rate. A protocol intending to balance power consumption from the remaining battery power of the node-based routing policy is proposed by Liang and Yang in [13]. The nodes with greater remaining power are allocated with more communication tasks. In [15], Kim et al. propose a lifetime-based routing strategy in which the survival time is estimated according to the residual power and current ratio of power consumption. The path with the longest node survival time is selected for data transmission.

Recently, limited studies in [19-21] are conducted on effective power saving strategies that specifically target at WNCS. Fischione et al. [20] propose a trade-off between wireless output power related to reliability and power consumption, where a physical characteristic model revealed quantitative relations with communication outage probability. They also focus on the lower layer optimal protocol design by considering the application layer requirements. Lino [21] discusses the optimal sleep mode control of wireless network nodes and proposes a trade-off method between control performance and power consumption. An optimal control strategy is applied to optimize the control period. In [22], event-predictive control for power saving of wireless networked control system is discussed. The key idea is to save power by maximizing the control interval with constrains of appropriate control performance. The proposed control method is rather complicated and requires online optimization mixed integer programming, which reduces the practicability. Thus, a simpler trade-off method for WNCS is required.

Power consumption, communication reliability, and system stability exist simultaneously and react with one another in wireless networked control systems. Supposed that the three factors are interdependent, most results achieved in wireless network power management and wired networked control systems cannot be directly applied to WNCS. Thus, the motivation of this paper is to find a bridge which can link the three factors and make a balance among these factors through the bridge parameter, such that the overall satisfactory performance can be achieved. Fortunately, the sampling period of sensor node is found to be the bridge parameter. From this point, a joint design method of adaptive sampling power efficiency algorithm and coordinated control method are discussed in this paper. An updating rule of sampling period is presented to satisfy the demands of wireless life span under constrains of network schedulability and control system stability. Convergence of the power efficiency algorithm is further proved. Subsequently, the control system is a varying-period system since the sampling periods of sensors are time-varying. It is then modeled as a class of switched control system with two types of behavior in each update period. The switched control law is applied to stabilize the control system and stability conditions are discussed. Also, the choosing rule of update period is given.

The remaining sections are organized as follows. Section 2 is the problem formulation. Section 3 presents the adaptive power efficiency algorithm. Section 4 discusses the coordinated wireless networked control system modeling and design method. Numerical simulation is given in Section 5. Section 6 is the conclusion of this paper.

\section{Problem Formulation}

2.1. Description of WNCSs. Consider the wireless networked control systems shown in Figure 1. There are three kinds of node in the system. Power consumption varies for the different kinds of node.

Besides, some necessary assumptions are made in this paper as the follows.

Assumption 1. The power of sensor and actuator nodes is supplied by battery while the power of controller is supplied by base station.

Assumption 2. The sensor and the actuator are clock driven while the controller is event driven. The sampling data is packed in one packet for transmission with time stamp.

Assumption 3. There exists transmission delay in the control loop, and it is assumed to be less than one sampling period.

\subsection{Analysis of Power Consumption in WNCSs. Sensor power} is consumed by three processes: data sampling, sample data reading by the $\mathrm{ADC}$, and data transfer. The power consumption of the controller node is also consumed by three processes: receiving data, calculating control variables, and sending data packet. The power of the actuator is consumed by two processes: receiving data and D/A conversion. The power consumption of different tasks is shown in Table 1 (see in [6]).

From the table, we get the following two conclusions:

(1) when the sensor transfers the same amount of data as the actuator receives, it will consume 2.5 times more power than the actuator will consume.

(2) data transfer consumes over $90 \%$ of the total sensor power consumption.

Given that the power required by control nodes can be supplied by the base station in most situations, the power required by the sensor nodes and actuators can be provided by batteries. Thus, sensors utilize the maximum amount 


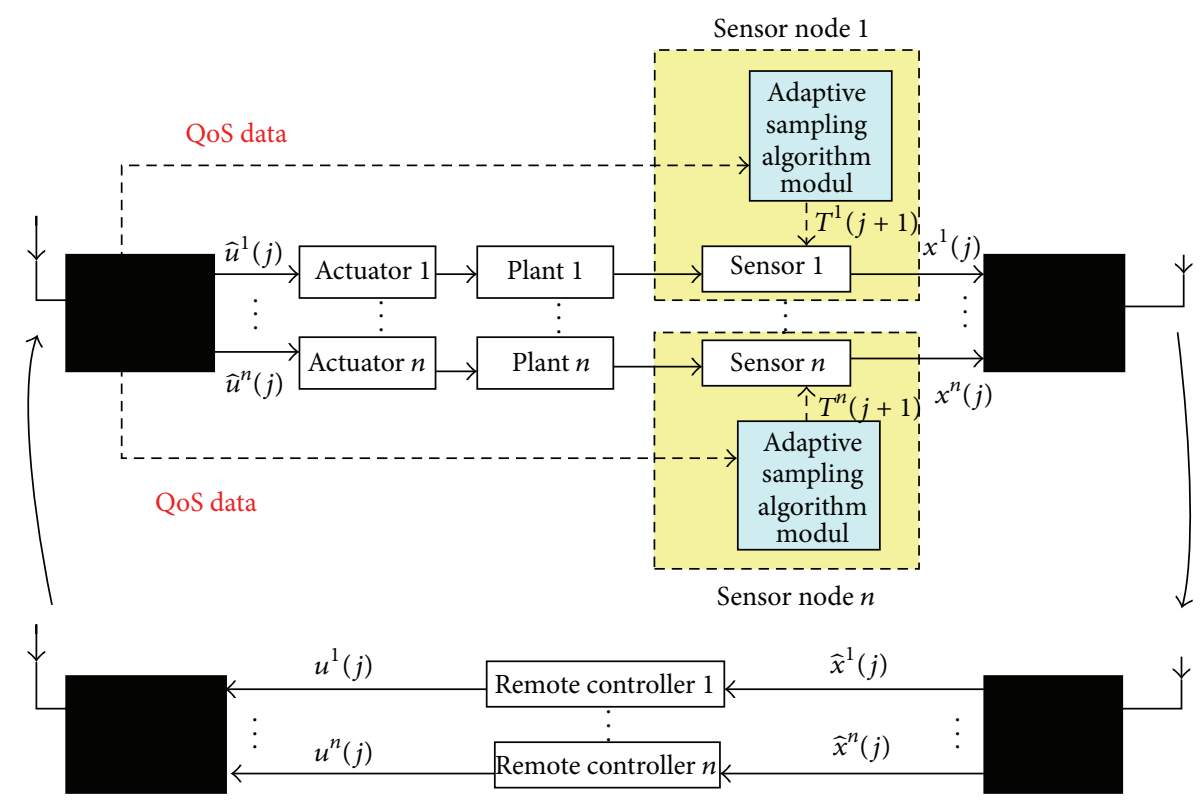

FIGURE 1: Structure of wireless networked control systems.

TABLE 1: Power consumption of different tasks.

\begin{tabular}{lc}
\hline Task & Energy consumption (nAh) \\
\hline Receive data & 8 \\
Transfer data & 20 \\
Read data & 0.011 \\
Sample data & 1.08 \\
\hline
\end{tabular}

of power consumption in WNCS. Managing the power consumption of sensors is the key to prolonging the survival time of the wireless network. A direct and effective method is to reduce as much of the transmission consumption as possible by properly adjusting the amount of sample data. This principle is the basis of our power control algorithm.

In wireless networks, the average power consumption of sending a packet can be described as [3]

$$
\bar{E}=b \times \text { packet_size }+c,
$$

where $b$ is the coefficient of power consumption and $c$ is the fixed power consumption of the node sending a data packet. According to Assumptions 1 and 2, a sensor node sends a packet to the corresponding controller at every sampling time. Given the lack of packet retransmission, the survival time of the sensor can be described as

$$
L=\sum_{i=1}^{\text {sum }}(t(i)-t(i-1)),
$$

where $L$ is the survival time of the sensor node, sum is the maximum number of packets sent by the sensor given an initial power, sum $=\left\lfloor E_{\text {init }} / \bar{E}\right\rfloor,\lfloor x\rfloor$ is the integral part of $x, E_{\text {init }}$ is the given initial power of the sensor, $t(i), i \in$ $\{1,2, \ldots$, sum $\}$ is the time that the sensor node sends the $i$ th data packet, and $t(0)$ is the initial time.

Survival time is dependent on transfer intervals. It can be prolonged by increasing the transfer intervals. Based on this premise as well as on the knowledge of the relationship between sampling period and control performance, we can cooperatively design the control and the network performances by adaptively adjusting the sampling period with a proper rule.

\section{Adaptive Sampling Power Efficiency Algorithm}

3.1. Update Rule of Sampling Period. For the consideration of simplicity and generality, we choose one of the control loops in the WNCS to describe the power control algorithm. Let $T^{\text {min }}$ be the lower bound of the sampling period to guarantee the schedulability of the network and let $T^{\max }$ be the upper bound of the sampling period to ensure system stability. Supposing that there are $M$ candidate sampling periods for choosing in the allowable range between the maximum and minimum bounds, $T_{l} \in\left[T^{\min }, T^{\max }\right], l=1,2, \ldots, M$. Furthermore, an update period $T_{M}, T_{M}>T^{\max }$ is designed for the sampling period renewal. The sampling period is renewed at each update instant as follows:

$$
\begin{aligned}
T(j+1) & =T(j)+\Delta T(j+1) \\
\Delta T(j+1) & =-\operatorname{sgn}\left(\widehat{L}(j)-L_{e}\right) \cdot \frac{S\left(T^{\mathrm{max}}-T^{\mathrm{min}}\right)}{M-1},
\end{aligned}
$$


where

$$
\begin{aligned}
S=\min \left\{\left\lfloor2(M-1)\left|\widehat{L}(j)-L_{e}\right|\right.\right. \\
\quad \times\left(\left(\frac{E_{\text {init }}}{\bar{E}}-\sum_{l=1}^{M} k_{l}(j) \frac{T_{M}}{T_{l}}-\frac{T_{M}}{T_{j}}\right)\right. \\
\left.\left.\left.\quad \times\left(T^{\max }-T^{\text {min }}\right)\right)^{-1}\right\rfloor, M-1\right\} ;
\end{aligned}
$$

$L_{e}$ is the demand of sensor survival time; $T(j)$ is the value of the sampling period in the $j$ th updating interval; $\operatorname{sgn}(x)$ is the signal of scalar $x ;\lfloor x\rfloor$ is the integral part of scalar $x ; k_{l}(j)$ is the number of updating intervals in which the sampling period is $T_{l}$ during the previous $j$ updates; $\widehat{L}(j)$ is the current predicted survival time of sensor node calculated by the following formula:

$$
\widehat{L}(j)=j T_{M}+\frac{E_{\text {rem }}(j)}{\bar{E}} \times T(j) ;
$$

$E_{\text {rem }}(j)$ is the current remaining power at the updating instant $j T_{M}$.

\subsection{Convergence of Power Efficiency Algorithm}

Theorem 1. For the WNCS described in Figure 1, considering the update rule of adaptive sampling period (3), if the minimum sampling period satisfies

$$
T^{\min }>\frac{L_{e}}{s u m},
$$

then the actual survival time of sensor node will reach its expected value $L_{e}$ through the proposed rule of sampling period update.

Proof. According to Formula (1), we have

$$
\widehat{L}(j+1)=(j+1) T_{M}+\frac{E_{\text {rem }}(j+1)}{\bar{E}} \times T(j+1) .
$$

The remaining power relationship at the two adjacent updating instants is given by

$$
E_{\text {rem }}(j+1)=E_{\text {rem }}(j)-\frac{T_{M}}{T(j)} \times \bar{E} .
$$

We assume that the sensor node has sampled the plant with the sampling period $T_{l}$ for $k_{l}(j)$ times from the initial to the current time instant. The remaining power can be calculated based on the initial power and consumed power:

$$
E_{\text {rem }}(j)=E_{\text {init }}-\sum_{l=1}^{M} k_{l}(j) \frac{T_{M} \bar{E}}{T_{l}} .
$$

Power control error is defined as $e(j)=\widehat{L}(j)-L_{e}$. According to formulas (7), (8), and (9), the dynamics of the error can be described as

$$
e(j+1)=e(j)+K(j) \Delta T(j+1),
$$

where $K(j)$ is defined as $K(j)=\operatorname{sum}-\sum_{l=1}^{M} k_{l}(j)\left(T_{M} / T_{l}\right)-$ $\left(T_{M} / T(j)\right)$. At the current time instant, $K(j)$ is a known variable.

The following Lyapunov function is introduced to prove convergence of the adaptive sampling power efficiency algorithm:

$$
V(j)=\frac{1}{2} e^{2}(j) .
$$

Considering Formula (10), it follows that

$$
\begin{aligned}
\Delta V(j) & =V(j+1)-V(j) \\
& =K(j) \Delta T(j+1) e(j)+\frac{1}{2} K^{2}(j) \Delta T^{2}(j+1) .
\end{aligned}
$$

With formula (3), we obtain

$$
\begin{array}{r}
\left.\begin{array}{r}
e(j)>0, \Delta T(j+1)<0 \\
e(j)<0, \Delta T(j+1)>0
\end{array}\right\} \Longrightarrow \Delta T(j+1) e(j)<0, \\
|\Delta T(j+1)||e(j)|>\frac{1}{2} K(j) \Delta T^{2}(j+1) .
\end{array}
$$

From inequalities (13), it can be concluded that $\Delta V(j)<$ 0 . Furthermore, to guarantee that the minimum survival time can reach the expected value, the minimum sampling period is bounded by $T^{\mathrm{min}} \geq L_{e} /$ sum. Consequently, the error system is stable, and the survival time can converge to the expected value if the conditions in Theorem 1 are satisfied.

Remark 2 (prediction of survival time). The actual survival time is unavailable at the current instant because the power consumption is time varying. However, it can be predicted by the known information of the remaining power and sampling period at the current instant. Formula (5) provides the prediction and indicates that the survival time of the node will be $\widehat{L}(j)$ if the sensor node maintains the sampling period $T(j)$ as unchanged from the current instant $t=j T_{M}$. The prediction of the survival time serves as a substitute for real survival time and is used to calculate the new sampling period.

Remark 3 (lower bound of sampling period). Taking IEEE $802.11 \mathrm{~b}$ as an example, the lower bound of the sampling period of sensor $T^{\text {min }}$ can be determined by the following formula:

$$
T^{\min }=\max \left\{\frac{L_{e}}{\operatorname{sum}}, \frac{S_{\mathrm{mc}} \times 2 \times N}{Q}\right\},
$$


where $\left(S_{\mathrm{mc}} \times 2 \times N\right) / Q$, is the allowable minimum sampling period when the wireless network can be schedulable (see in [6]), and

$$
\begin{gathered}
Q=\frac{S_{\mathrm{mc}}}{T_{\mathrm{DIFS}}+\left(C W_{\mathrm{min}} \times T_{\mathrm{SIFS}} / 2\right)+T_{\text {frame }}+T_{\mathrm{SIFS}}+T_{\mathrm{ACK}}} \\
T_{\text {frame }}=\frac{S_{\mathrm{PHY}}}{R_{l}}+\frac{S_{\mathrm{MAC}}+S_{\mathrm{mc}}}{R_{t}} \\
T_{\text {frame }}=\frac{S_{\mathrm{PHY}}}{R_{l}}+\frac{S_{\mathrm{MAC}}+S_{\mathrm{mc}}}{R_{t}} .
\end{gathered}
$$

$R_{t}$ is the transmission rate, $R_{l}$ is the legacy transmission rate, $S_{\mathrm{mc}}$ is the measurement-control data size, $S_{\mathrm{PHY}}$ is the size of control frame in physical layer, and $S_{\mathrm{MAC}}$ is the data size of ACK and is a confirmed sign in the header of TCP data packet that confirms the received TCP message. $T_{\text {SIFS }}$ is the shortest time period of the $802.11 \mathrm{~b}$ protocol for the interval of frames requiring immediate response. $T_{\mathrm{DIFS}}$ is the time segment for the interval of the time frame of the distributed coordination function for sending in IEEE 802.11b. $T_{\text {PIFS }}$ is the time segment for the interval of the time frame of the centralized coordination function for sending, which satisfies

$$
T_{\mathrm{PIFS}}=T_{\mathrm{DIFS}}-T_{\text {slot }}, \quad T_{\text {slot }}=T_{\mathrm{PIFS}}-T_{\mathrm{SIFS}} .
$$

$\mathrm{CW}$ is the contention window. Wireless network parameters under the $802.11 \mathrm{~b}$ direct sequence spread spectrum are shown in Table 2.

Remark 4 (upper bound of sampling period). For a SISO system, the maximum sampling period can be obtained using Shannon sampling theorem. For a MIMO system, the following method can be used to obtain the upper bound. If the system feedback control law is given ahead, then $T^{\max }$ can be obtained by solving the following optimal problem:

$$
\begin{array}{ll}
\max & T \\
\text { s.t } & \left\|\operatorname{eig}\left(e^{\mathbf{A} T}+\int_{0}^{T} e^{\mathbf{A} T} d t \mathbf{B} \mathbf{K}_{0}\right)\right\|<1,
\end{array}
$$

where $\mathbf{A}$ is the system matrix and $\mathbf{B}$ is the control input matrix. $\mathbf{K}_{0}$ is the Kalman gain, which satisfies

$$
\boldsymbol{\operatorname { R e }}\left(\operatorname{eig}\left(\mathbf{A}+\mathbf{B K}_{0}\right)\right)<0,
$$

where $\operatorname{Re}(v)$ denotes the real part of $v$ and $\operatorname{eig}(\mathbf{X})$ denotes the eigenvalues of matrix $\mathbf{X}$. Gain $\mathbf{K}_{0}$ can be determined by the pole assignment in the continuous time domain.

However, the above optimal problem is difficult to solve directly. The following iteration method can be used to obtain the approximate optimal value of $T^{\max }$ :

Step 1. Let $q=1$, and the initial value of $T^{\max }$ is $T^{\max }(q)=$ $2 T^{\mathrm{min}}$. If the condition can satisfy the constraints of the optimal problem, go to Step 2; or else, go to Step 3.
TABLE 2: Wireless network parameters under $802.11 \mathrm{~b}$ direct sequence spread spectrum.

\begin{tabular}{lc}
\hline Parameter & Value \\
\hline$R_{t}$ & $11 \mathrm{Mbps}$ \\
$R_{l}$ & $1 \mathrm{Mbps}$ \\
$T_{\mathrm{SIFS}}, T_{\mathrm{DIFS}}, T_{\text {slot }}$ & $10,50,20$ us \\
$S_{\mathrm{MAC}}, S_{\mathrm{PHY}}$ & 34,24 bytes \\
$S_{\mathrm{mc}}$ & 80 bytes \\
$S_{\mathrm{ACK}}$ & 14 bytes + PHY header \\
$\mathrm{CW}_{\min }, \mathrm{CW}_{\max }$ & 32,1024 \\
\hline
\end{tabular}

Step 2. Let $q=q+1$, and let $T^{\max }(q)=2 T^{\max }(q-1)$. If the condition can fulfill the constraints of the optimal problem, cycle Step 2; or else, end the iteration and then let $T^{\max }=$ $T^{\max }(q-1)$.

Step 3. Let $T^{\max }(q)=\left(1+(3 / 4)^{q}\right) T^{\min }$. If the condition still does not satisfy the constraints of the optimal problem, let $q=q+1$ and cycle Step 3; or else end the iteration and let $T^{\max }=T^{\max }(q)$.

\section{Modeling and Stability Analysis of Adaptive Sampling Period WNCS}

4.1. Modeling of Adaptive Sampling Period WNCS. Considering the generality, one of the control loops in the WNCS is chosen as an example to illustrate the modeling approach. For the WNCS power consumption managed by algorithm in Section 2, the dynamics of the control system is time-varying. Since the sampling period varies among the $M$ candidates, the system can be considered a switched system with $M$ modes from the perspective of the switched control system scheme. Each switching mode is corresponded to one of the candidates. According to Assumption 1, the sensor and actuator nodes are assumed to be clock-driven. It results that the switching occurs at some of the sampling instants. Additionally, according to Assumption 3, the inevitable existence of network-induced delay is taken into account and it is less than one sampling period.

We consider a plant of control loop $i$ in the WNCS with the following dynamics:

$$
\begin{aligned}
& \dot{x}^{i}(t)=\mathbf{A}^{i} x^{i}(t)+\mathbf{B}^{i} u^{i}(t) \\
& y^{i}(t)=\mathbf{C}^{i} x^{i}(t)
\end{aligned}
$$

where $x^{i}(t) \in \mathbf{R}^{n}$ is the plant state, $u^{i}(t) \in \mathbf{R}^{m}$ is the control input, and $y^{i}(t) \in \mathbf{R}^{p}$ is the plant output. $\mathbf{A}^{i} \in \mathbf{R}^{n \times n}, \mathbf{B}^{i} \in$ $\mathbf{R}^{n \times m}$, and $\mathbf{C}^{i} \in \mathbf{R}^{p \times n}$ are the matrices of state, control input, and output matrices, respectively. Due to the generality of $i$, we omit the superscript $i$ in the following model description and deduction.

Discretizing system (20) with sampling rate $T_{l}$ and considering network-induced delay less than one sampling 


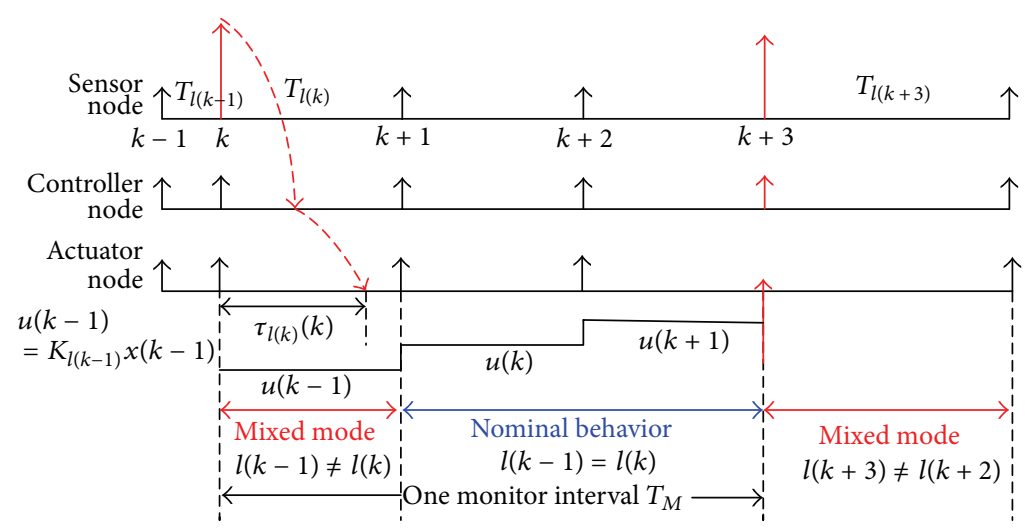

FIGURE 2: Evolution over one period of switched WNCS with two types of behavior.

period, the discrete dynamics of the open control loop can be described as

$$
\begin{gathered}
x(k+1)=\boldsymbol{\Phi}_{l(k)} x(k)+\Gamma_{l(k)} u(k-1) \\
y(k)=\mathbf{C} x(k),
\end{gathered}
$$

where $\boldsymbol{\Phi}_{l(k)}=e^{\mathbf{A} T_{l(k)}} ; \boldsymbol{\Gamma}_{l}=\int_{0}^{T_{l(k)}} e^{\mathbf{A} t} d t \mathbf{B}$; and $l(k)$ is the identification of sampling period at the $k$ th sampling instant, $l(k) \in$ $\mathbb{Z} \rightarrow \ell=\{0,1, \ldots, M-1\}$.

For the discrete switched system (21), a switched state feedback controller is introduced in the following form:

$$
u(k)=\mathbf{K}_{\gamma(k)} x(k),
$$

where $\gamma(k) \in \mathbb{Z} \rightarrow \ell=\{0,1, \ldots, M-1\}$ denotes the switching signal used in the control.

Consequently, the closed-loop WNCS can be written as

$$
\left[\begin{array}{c}
x(k+1) \\
x(k)
\end{array}\right]=\left[\begin{array}{cc}
\boldsymbol{\Phi}_{l(k)} & \boldsymbol{\Gamma}_{l(k)} \mathbf{K}_{\gamma(k)} \\
\mathbf{I} & 0
\end{array}\right]\left[\begin{array}{c}
x(k) \\
x(k-1)
\end{array}\right] .
$$

\subsection{Stability Analysis of Adaptive Sampling Period WNCS.} The control gains $\mathbf{K}_{\gamma(k)}$ are assumed to be designed in such a way that the closed-loop system is asymptotically stable when $\gamma(k)=l(k)$. Ideally, the switching signal used in control $\gamma(k)$ is the same as the real signal $l(k)$. However, this view is unrealistic in WNCS with network-induced delay, where $\gamma(k)=l(k-1)$. The evolution over one sampling period can be described by two distinct types of behavior: the nominal and mixed mode sampling periods, as shown in Figure 2.

(1) Nominal sampling period is when the system evolution uses the right switching information:

$$
\gamma(k)=l(k-1), \quad l(k)=l(k-1) .
$$

(2) Mixed mode sampling period is when the system command uses a wrong feedback gain

$$
\gamma(k)=l(k-1), \quad l(k) \neq l(k-1) .
$$

Denoting $\Delta^{m}$ as the samples spent in the mixed mode, since the delay is less than one sampling period, it follows that
$\Delta^{m}$ has a range of $0 \leq \Delta^{m} \leq 1$. Moreover, we assume that the system is controlled using the right gains for at least $\Delta^{n}$ samples before another switching occurs.

The next thing we should do is to guarantee the closedloop system remaining stable with the designed state feedback control gains when the switching signal is temporarily uncertain. Consider the scalars $\theta_{n}>0, \theta_{m}>0$ and the symmetric positive definite matrices $\mathbf{P}_{l}^{n}, \mathbf{P}_{(l, \gamma)}^{m}$ with $(l, \gamma) \in$ $\ell \times \ell$, which satisfy the following matrix inequalities:

$$
\begin{gathered}
\overline{\boldsymbol{\Phi}}_{(l, \gamma)}^{T}\left(\mathbf{P}_{(l, \gamma)}^{m}\right)^{-1} \overline{\boldsymbol{\Phi}}_{(l, \gamma)}-\theta_{m} \mathbf{P}_{(l, \gamma)}^{m}<0, \quad \forall(l, \gamma) \in \ell \times \ell \\
\overline{\boldsymbol{\Phi}}_{(l, l)}^{T} \mathbf{P}_{l}^{n} \overline{\boldsymbol{\Phi}}_{(l, l)}-\theta_{n} \mathbf{P}_{l}^{n}<0, \quad \forall l \in \ell .
\end{gathered}
$$

Moreover, consider the following two scalars,

$$
\begin{aligned}
& \beta_{n}=\frac{\max _{l \in \ell} e \operatorname{eig}_{\max }\left(\mathbf{P}_{l}^{n}\right)}{\min _{l \in \ell} \operatorname{eig}{ }_{\min }\left(\mathbf{P}_{l}^{n}\right)}, \\
& \beta_{m}=\frac{\max _{(l, \gamma) \in \ell \times \ell} \operatorname{eig} g_{\max }\left(\mathbf{P}_{(l, \gamma)}^{m}\right)}{\min _{(l, \gamma) \in \ell \times \ell} \operatorname{eig} g_{\text {min }}\left(\mathbf{P}_{(l, \gamma)}^{m}\right)},
\end{aligned}
$$

where eig $\max _{\max }(\mathbf{X})$ and eig $\min _{\text {min }}(\mathbf{X})$ denote the maximum and the minimum eigenvalues of matrix $\mathbf{X}$, respectively.

Then, the stability of the closed-loop WNCS (23) can be guaranteed by the following theorem.

Theorem 5. Let $\theta_{n}^{*}, \theta_{m}^{*}$ be the solutions of the optimization problems $\theta_{n}^{*}=\min \theta_{n}$ and $\theta_{m}^{*}=\min \theta_{m}$ subject to matrix inequalities (26). Closed-loop system (23) is asymptotically stable if

$$
\beta_{n} \cdot \beta_{m} \cdot\left(\theta_{n}^{*}\right)^{\Delta^{n}} \cdot \theta_{m}^{*}<1 .
$$

Proof. We consider the following Lyapunov functions:

$$
V^{n}(k)=\bar{x}^{T}(k) \mathbf{P}_{l}^{n} \bar{x}(k), \quad V^{m}(k)=\bar{x}^{T}(k) \mathbf{P}_{(l, \gamma)}^{m} \bar{x}(k) .
$$

Inequalities (13) show that

$$
V^{n}\left(k_{1}\right)<\left(\theta_{n}^{*}\right)^{k_{1}-k_{0}} V^{n}\left(k_{0}\right) .
$$


$\forall k \in\left[k_{0}, k_{1}\right)$, if the right switching signal is used in the control, $l(k)=\gamma(k)=l\left(k_{0}\right)$, then

$$
V^{m}\left(k_{1}\right)<\left(\theta_{m}^{*}\right)^{k_{1}-k_{0}} V^{m}\left(k_{0}\right) \text {. }
$$

$\forall k \in\left[k_{0}, k_{1}\right)$, if the switching signal in the control is not necessarily the same as the real signal, the pair $l(k), \gamma(k)$ takes an arbitrary value $l\left(k_{0}\right), \gamma\left(k_{0}\right)$ in $\ell \times \ell$.

Since the controller gains $\mathbf{K}_{l}$ are designed to make the matrices $\overline{\boldsymbol{\Phi}}_{(l, l)}$ stable, the scalar $\theta_{n}$ is smaller than one, $\theta_{n}<1$. The scalar $\theta_{m}$ may be greater than one, $\theta_{m}>1$, since the gains $\mathbf{K}_{\gamma}$ are not designed to stabilize combinations other than $\overline{\boldsymbol{\Phi}}_{(\gamma, \gamma)}$.

Combining inequalities (26) yield

$$
\begin{aligned}
& \min _{l \in \ell} \operatorname{eig}_{\min }\left(\mathbf{P}_{l}^{n}\right)\left\|\bar{x}\left(k_{1}\right)\right\|^{2} \\
& \quad<\left(\theta_{n}^{*}\right)^{k_{1}-k_{0}} \operatorname{maxeig}_{l \in \ell}\left(\mathbf{P}_{l}^{n}\right)\left\|\bar{x}\left(k_{0}\right)\right\|^{2} \quad \forall l \in \ell \\
& \min _{(l, \gamma) \in \ell \times \ell} \operatorname{eig}_{\min }\left(\mathbf{P}_{(l, \gamma)}^{m}\right)\left\|\bar{x}\left(k_{1}\right)\right\|^{2} \\
& \quad<\max _{(l, \gamma) \in \ell \times \ell} \operatorname{eig}_{\max }\left(\mathbf{P}_{(l, \gamma)}^{m}\right)\left\|\bar{x}\left(k_{0}\right)\right\|^{2} \quad \forall(l, \gamma) \in \ell \times \ell .
\end{aligned}
$$

With the definitions of $\beta_{n}$ and $\beta_{m}$, we can obtain the state vector norm decay or growth rate in a nominal regime and in an uncertain switching signal regime as follows:

$$
\begin{gathered}
\left\|\bar{x}\left(k_{1}\right)\right\|^{2}<\beta_{n} \cdot\left(\theta_{n}^{*}\right)^{k_{1}-k_{0}}\left\|\bar{x}\left(k_{0}\right)\right\|^{2}, \quad \forall l \in \ell \\
\left\|\bar{x}\left(k_{1}\right)\right\|^{2}<\beta_{m} \cdot\left(\theta_{m}^{*}\right)^{k_{1}-k_{0}}\left\|\bar{x}\left(k_{0}\right)\right\|^{2}, \quad \forall(l, \gamma) \in \ell \times \ell .
\end{gathered}
$$

Let $k_{s}^{m}$ describe the instants when the closed-loop system jumps to a mixed mode with uncertain switching signal, and let $k_{s}^{n}$ be the instance when the system enters into a normal regime. With definitions and bounds of $\Delta^{m}$ and $\Delta^{n}$, it follows that

$$
0 \leq \Delta^{m}=k_{s}^{n}-k_{s}^{m} \leq 1, \quad k_{s+1}^{m}-k_{s}^{n} \leq \Delta^{n} .
$$

Without loss of generality, we assume that the system starts with a mixed-mode behavior, $k_{s}^{m}<k_{s}^{n}$. The system behavior in time interval $k \in\left[k_{s}^{m}, k_{s+1}^{m}\right)$ is then analyzed.

Given that $l(k) \neq \gamma(k), \forall k \in\left[k_{s}^{m}, k_{s}^{n}\right)$ and $l(k)=$ $\gamma(k), \forall k \in\left[k_{s}^{n}, k_{s+1}^{m}\right)$, using inequalities (26), the norm of the state at the end of the sequence can be upper bounded asd

$$
\begin{aligned}
\left\|\bar{x}\left(k_{s+1}^{m}\right)\right\|^{2} & <\beta_{m} \cdot \beta_{n} \cdot\left(\theta_{m}^{*}\right)^{k_{s}^{n}-k_{s}^{u}} \cdot\left(\theta_{n}^{*}\right)^{k_{s+1}^{m}-k_{s}^{n}}\left\|\bar{x}\left(k_{s}^{m}\right)\right\|^{2} \\
& <\left\|\bar{x}\left(k_{s+1}^{m}\right)\right\|^{2}<\beta_{m} \cdot \beta_{n} \cdot \theta_{m}^{*} \cdot\left(\theta_{n}^{*}\right)^{\Delta^{n}}\left\|\bar{x}\left(k_{s}^{m}\right)\right\|^{2} .
\end{aligned}
$$

It indicates that closed loop (16) will be asymptotically stable if condition (28) in Theorem 5 is satisfied.

\subsection{Choosing Rule of Update Period $T_{M}$}

Theorem 6. Consider the WNCS with adaptive sampling period rule (3). The WNCS can be stabilized by the switched state feedback controllers (22), whereas the survival time can meet its expected value $L_{e}$, if the update period $T_{M}$ satisfies the following conditions:

$$
\begin{aligned}
& \text { (1) } T_{M} \geq \max \left(\Delta_{i}^{n}+1\right)\left[T_{1}^{\max }, T_{2}^{\max }, \ldots, T_{n}^{\max }\right] \\
& \text { (2) }\left\lfloor\frac{L_{e}}{T_{M}}\right\rfloor \cdot T_{M} \leq \frac{E_{\text {init }}}{\bar{E}} \min \left\{T_{1}^{\min }, T_{2}^{\min }, \ldots, T_{n}^{\min }\right\},
\end{aligned}
$$

where $\Delta_{i}^{n}$ is the least nominal sampling period of control loop $i$ and the solution of Theorem $5,\left[T_{1}^{\max }, T_{2}^{\max }, \ldots, T_{n}^{\max }\right]$, is the least common multiple of the maximum sampling period for all the $n$ control loops in the WNCS.

Proof. According to Theorem 5, control loop $i$ in the WNCS should stay at least $\Delta_{i}^{n}$ in a nominal sampling period in one updating interval for stability. With the consideration of $0 \leq$ $\Delta_{i}^{m} \leq 1$, if the update period satisfies condition (1), all control loops in the WNCS will meet the demands of their least nominal sampling periods and will be stable. As a complete system, WNCS is composed of $n$ control loops that will be stable when condition (1) is satisfied.

Condition (2) in Theorem 6 provides the upper bound of the updating period. For the power efficiency algorithm in Theorem 1, with the definition of $k_{l}(j)$, there exists

$$
\sum_{l=1}^{M} k_{l}(j) \frac{T_{M}}{T_{l}}+\frac{T_{M}}{T_{j}} \leq\left\lfloor\frac{L_{e}}{T_{M}}\right\rfloor \cdot \frac{T_{M}}{\min \left\{T_{1}^{\min }, T_{2}^{\min }, \ldots, T_{n}^{\min }\right\}} .
$$

If condition (2) in Theorem 6 is satisfied, the item $\left(\left(E_{\text {init }} / \bar{E}\right)-\right.$ $\left.\sum_{l=1}^{M} k_{l}(j)\left(T_{M} / T_{l}\right)-\left(T_{M} / T_{j}\right)\right)$ in the denominator of formula (3) will be greater than zero, which guarantees that formula (3) has physical meaning and is solvable. The survival time will, thus, reach the expected value by applying the power efficiency algorithm.

\section{Numerical Example}

Simulation studies are performed on a WNCS closed by an IEEE 802.11b wireless network with two control loops sharing the network resources. The two control loops are assumed to have the same dynamics but with different initial conditions:

$$
\begin{array}{r}
\dot{x}^{i}(t)=\left[\begin{array}{cc}
-1 & -0.1 \\
0 & 0.95
\end{array}\right] x^{i}(t)+\left[\begin{array}{l}
-0.15 \\
-0.43
\end{array}\right] u^{i}(t) \\
y^{i}(t)=\left[\begin{array}{ll}
1 & 1
\end{array}\right] x^{i}(t), \\
i=1,2 \\
x^{1}(0)=\left[\begin{array}{ll}
-5 & 5
\end{array}\right]^{T}, \quad x^{2}(0)=\left[\begin{array}{ll}
-5 & 10
\end{array}\right]^{T} .
\end{array}
$$

The wireless network parameters are set as in Table 2. The time delays in both loops are less than one sampling period. Some other necessary parameters are given as shown in Table 2.

In Table 3, the minimum and maximum sampling periods are computed by the methods in Section 3. Solving 
TABLE 3: Simulation parameters.

\begin{tabular}{lc}
\hline Parameter & Value \\
\hline Initial energy of both sensors $E_{\text {init }}$ & $0.15 \mathrm{~J}$ \\
Sensor 1 expected survival time $L_{e 1}$ & $75 \mathrm{~s}$ \\
Sensor 2 expected survival time $L_{e 2}$ & $70 \mathrm{~s}$ \\
Unit transmission energy $\bar{E}$ & $25 \mathrm{dbm}$ \\
Number of sampling period candidates $M$ & 10 \\
The minimum sampling period of loop $1 T_{1}^{\min }$ & $1 \mathrm{~ms}$ \\
The maximum sampling period of loop1 $T_{1}^{\max }$ & $256 \mathrm{~ms}$ \\
The minimum sampling period of loop $2 T_{2}^{\min }$ & $1 \mathrm{~ms}$ \\
The maximum sampling period of loop2 $T_{2}^{\max }$ & $256 \mathrm{~ms}$ \\
\hline
\end{tabular}

TABLE 4: Controller gains of ten sampling modes.

\begin{tabular}{lc}
\hline Sampling period $(/ \mathrm{ms})$ & Controller gain \\
\hline$T_{0}=1$ & $K_{0}=\left[\begin{array}{ll}1117.6 & 1118.8\end{array}\right]$ \\
$T_{1}=29$ & $K_{1}=\left[\begin{array}{ll}39.4837 & 40.5512\end{array}\right]$ \\
$T_{2}=58$ & $K_{2}=\left[\begin{array}{ll}19.4678 & 20.4319\end{array}\right]$ \\
$T_{3}=86$ & $K_{3}=\left[\begin{array}{ll}12.5668 & 13.4279\end{array}\right]$ \\
$T_{4}=114$ & $K_{4}=\left[\begin{array}{ll}9.0353 & 9.7947\end{array}\right]$ \\
$T_{5}=143$ & $K_{5}=\left[\begin{array}{ll}6.8663 & 7.5265\end{array}\right]$ \\
$T_{6}=171$ & $K_{6}=\left[\begin{array}{ll}5.383 & 5.947\end{array}\right]$ \\
$T_{7}=199$ & $K_{7}=\left[\begin{array}{ll}4.295 & 4.769\end{array}\right]$ \\
$T_{8}=227$ & $K_{8}=\left[\begin{array}{ll}4.225 & 3.852\end{array}\right]$ \\
$T_{9}=256$ & $K_{9}=\left[\begin{array}{ll}3.707 & 2.95\end{array}\right]$ \\
\hline
\end{tabular}

inequality (28) in Theorem 5 , it yields $\Delta_{1}^{n}=\Delta_{2}^{n}=8, \Delta^{m}=1$. Also, the update period can be chosen by Theorem 6 as $T_{M}=$ $150 \mathrm{~ms}$.

Solving matrix inequalities (18) and (19), the controller gains of ten switching modes can be obtained as in Table 4 .

With the above simulation parameters and controller gains, the curves of survival time prediction, power consumption, and control output of both control loops are shown in Figures 3 to 8 .

Analyzing the simulation curves, we have the following results.

(1) Figures 3, 6, 4, and 7 imply that both sensor 1 and sensor 2 can meet their expected survival time requirements.

(2) The power consumption in three cases of minimum sampling, maximum sampling, and the proposed adaptive sampling is compared in Figures 4 and 7. It is obvious that the power is consumed much faster than the other two cases. In the case of the adaptive sampling, the power consumption varies according to both the requirements of the control performance and survival time. At the beginning, the power consumption curves vary quickly and more power are consumed because of the control systems not reaching stable yet. Then, after the control systems

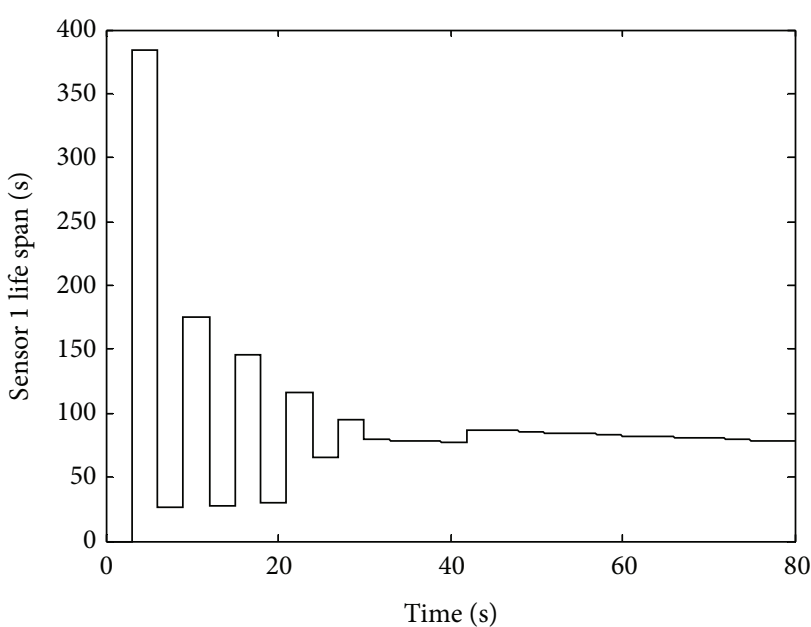

FIGURE 3: Sensor 1 survival time prediction.

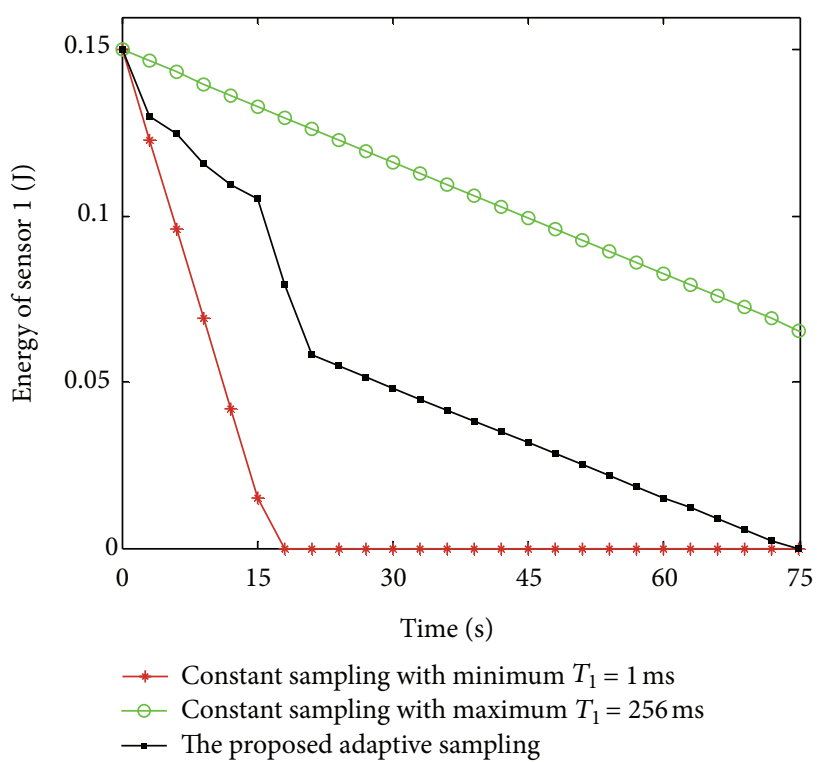

FIGURE 4: Sensor 1 power consumption comparison in three cases.

are settled, it tends to the minimum consumption rate which is corresponding to the maximum sampling period.

(3) Figures 5 and 8 are the control outputs of loop1 and loop 2 in the three cases mentioned above. The figures show that the control systems can be stabilized through the proposed joint design methods. In three cases, the adaptive sampling can get the control performances closed to the case of minimum sampling.

(4) Combining Figures 4, 5, 7, and 8, it can be concluded that the proposed joint design method achieves a tradeoff between the performances of control and power efficiency. 


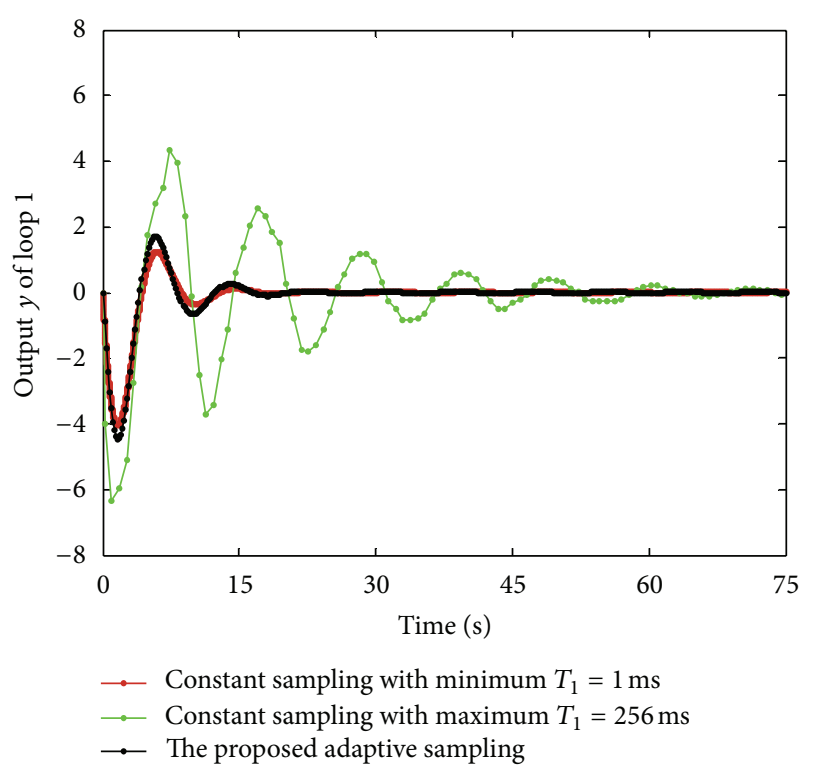

FIGURE 5: Control loop1 output comparison in three cases.

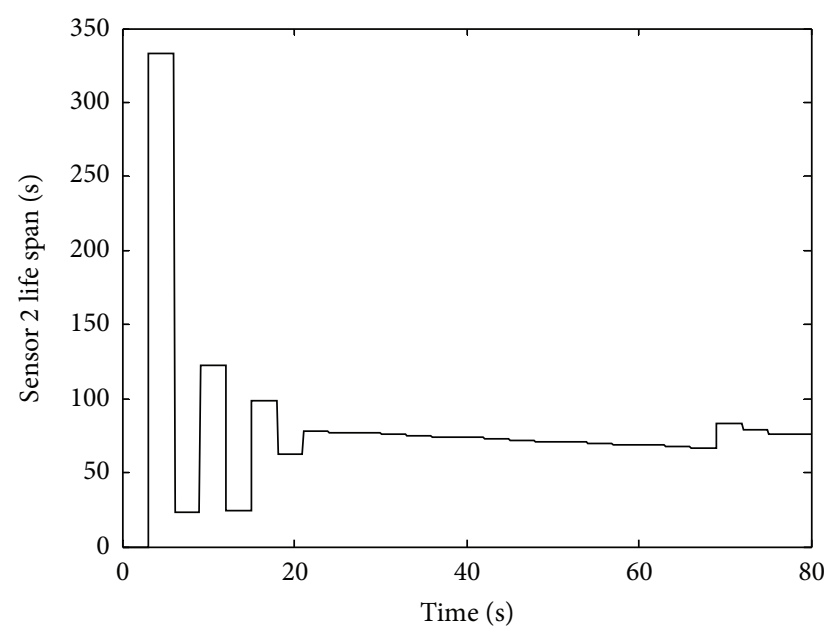

FIGURE 6: Sensor 2 survival time prediction.

\section{Conclusion}

This paper presents a joint design method for wireless networked control systems with limited power constraint. A power efficiency algorithm based on the adaptive sampling period is put forward to satisfy the demands of sensor survival time and system stability. Then, the time-varying control system with transmission delay is modeled as a switched system with uncertain switching signals. A dwelltime-dependent control method is discussed to guarantee the stability of WNCS. Simulation results show the effectiveness of the proposed method and indicate that it can achieve good tradeoff performance. Methods by which to reduce power consumption from the aspect of a single node as well as balancing power consumption from the global network perspective are worthy of further exploration.

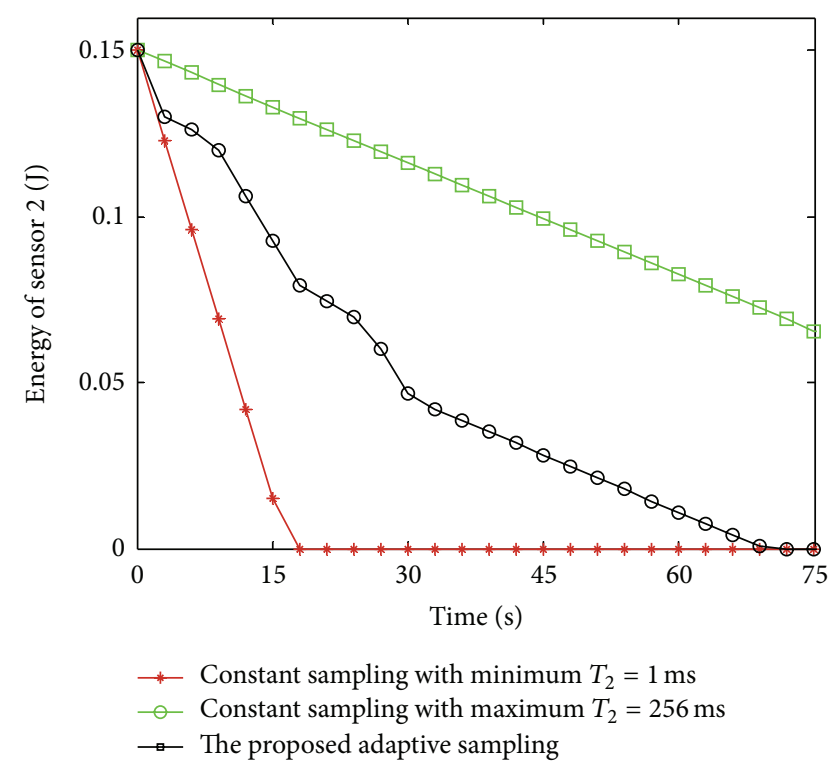

FIGURE 7: Sensor 2 power consumption comparison in three cases.

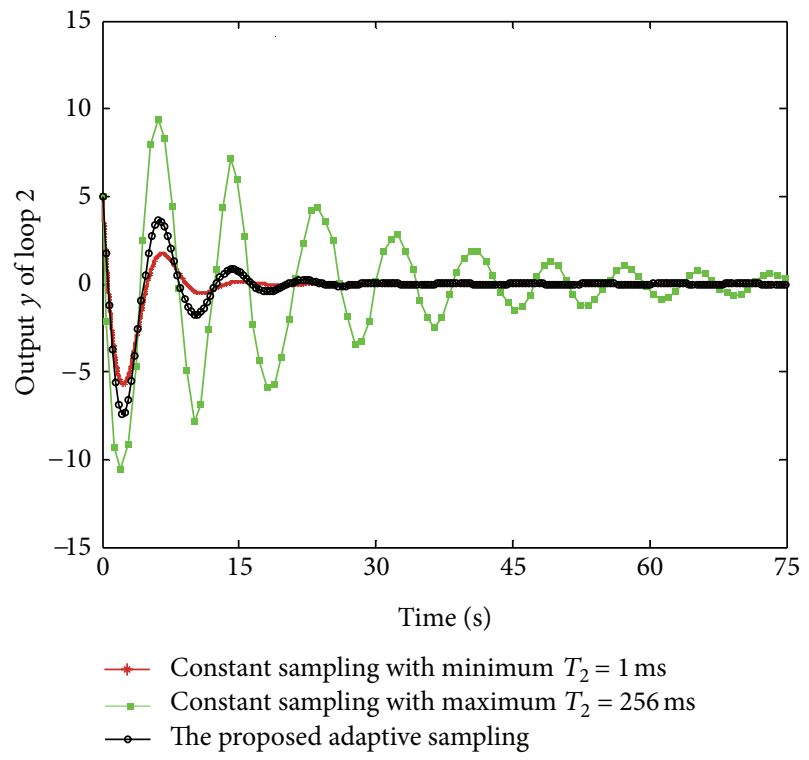

Figure 8: Control loop2 output comparison in three cases.

\section{Conflict of Interests}

The authors declare that there is no conflict of interests regarding the publication of this paper.

\section{Acknowledgments}

The working is sponsored by The NSFC (no. 61202473) and The Natural Science Foundation of Jiangsu Province (no. BK2012551). 


\section{References}

[1] W. Dieterle, H. D. Kochs, and E. Dittmar, "Communication architectures for distributed computer control systems," Control Engineering Practice, vol. 3, no. 8, pp. 1171-1176, 1995.

[2] H. A. Thompson, "Wireless and Internet communications technologies for monitoring and control," Control Engineering Practice, vol. 12, no. 6, pp. 781-791, 2004.

[3] P. R. Kumar, "New technological vistas for systems and control," IEEE Control Systems Magazine, vol. 21, no. 1, pp. 24-37, 2001.

[4] X. Liu and A. Goldsmith, "Wireless medium access control in networked control systems," in Proceedings of the American Control Conference (AAC '04), pp. 3605-3610, Boston, Mass, USA, July 2004.

[5] J. Colandairaj, G. W. Irwin, and W. G. Scanlon, "Wireless networked control systems with QoS-based sampling," IET Control Theory and Applications, vol. 1, no. 1, pp. 430-438, 2007.

[6] N. Israr, W. G. Scanlon, and G. W. Irwin, "A cross-layer communication framework for wireless networked control systems," in Proceedings of the 1st International Conference on Wireless Communication, Vehicular Technology, Information Theory and Aerospace and Electronic Systems Technology (VITAE '09), pp. 577-581, Aalborg, Denmark, May 2009.

[7] S. Dai, H. Lin, and S. S. Ge, "Scheduling-and-control codesign for a collection of networked control systems with uncertain delays," IEEE Transactions on Control Systems Technology, vol. 18, no. 1, pp. 66-78, 2010.

[8] A. V. Savkin, "Analysis and synthesis of networked control systems: topological entropy, observability, robustness and optimal control," Automatica, vol. 42, no. 1, pp. 51-62, 2006.

[9] J. Z. Luo, F. Dong, and J. X. Cao, "A novel task scheduling algorithm based on dynamic critical path and effective duplication for pervasive computing environment," Wireless Communications \& Mobile Computing, vol. 10, no. 10, pp. 12831302, 2010.

[10] X. Yin, X. Zhou, Z. Li, and S. Li, "Jint rate control and power control for lifetime maximization in Wreless Sensor Ntworks," Journal of Internet Technology, vol. 12, no. 1, pp. 69-78, 2011.

[11] M. Pajic, S. Sundaram, G. J. Pappas, and R. Mangharam, "The wireless control network: a new approach for control over networks," IEEE Transactions on Automatic Control, vol. 56, no. 10, pp. 2305-2318, 2011.

[12] J. Colandairaj, G. W. Irwin, and W. G. Scanlon, "A co-design solution for wireless feedback control," in Proceeding of the International Conference on Networking, Sensing and Control (ICNSC '07), pp. 404-409, London, UK, April 2007.

[13] W. Liang and Y. Yang, "Maximizing battery life routing in wireless ad hoc networks," in Proceedings of the 37th International Conference on System Sciences, pp. 4739-4746, IEEE, Honolulu, Hawaii, USA, January 2004.

[14] K. Brian, J. Haberman, and W. Sheppard, Overlapping Particle Swarms for Energy-Efficient Routing in Sensor Networks, Wireless Network, Springer, 2011.

[15] D. Kim, K. Dantu, and M. Pedram, "Lifetime prediction routing in mobile Ad Hoc networks," in Proceedings of the IEEE Wireless Communication and Networking Conference, New York, NY, USA, 2003.

[16] L. Hetel, J. Daafouz, and C. Iung, "Stability analysis for discrete time switched systems with temporary uncertain switching signal," in Proceedings of the 46th IEEE Conference on Decision and Control (CDC '07), pp. 5623-5628, New Orleans, Fla, USA, December 2007.
[17] S. Limin, Wireless Sensor Network, Tsinghua University Press, Beijing, China, 2006.

[18] Y. He, I. Lee, and L. Guan, "Distributed algorithms for network lifetime maximization in wireless visual sensor networks," IEEE Transactions on Circuits and Systems for Video Technology, vol. 19, no. 5, pp. 704-718, 2009.

[19] B. A. Bakr and L. Lilien, "A quantitative comparison of energy consumption and WSN lifetime for LEACH and LEACHSM," in Proceedings of the 31st International Conference on Distributed Computing Systems Workshops (ICDCSW '11), pp. 182-191, Minneapolis, Minn, USA, June 2011.

[20] C. Fischione, A. Bonivento, A. Sangiovanni-Vincentelli, F. Santucci, and K. H. Johansson, "Performance analysis of collaborative spatio-temporal processing for wireless sensor networks," in Proceedings of the 3rd IEEE Consumer Communications and Networking Conference (CCNC '06), pp. 325-329, Las Vegas, Nev, USA, January 2006.

[21] Y. Iino, "Some considerations of wireless sensor network based control systems," in Proceedings of the SICE Conference on Control Systems (CCS '07), Tokyo, Japan, 2007.

[22] Y. Iino, T. Hatanaka, and M. Fujita, "Event-predictive control for energy saving of wireless networked control system," in Proceedings of the American Control Conference (ACC '09), pp. 2236-2242, St. Louis, Mo, USA, June 2009. 


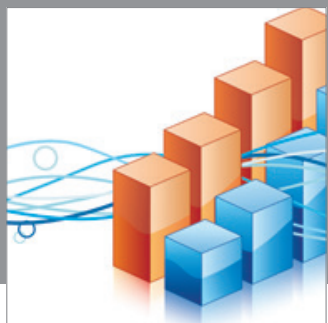

Advances in

Operations Research

mansans

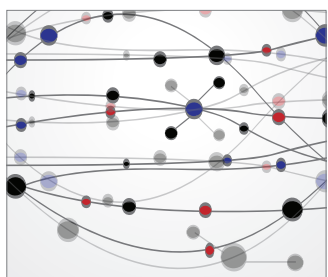

The Scientific World Journal
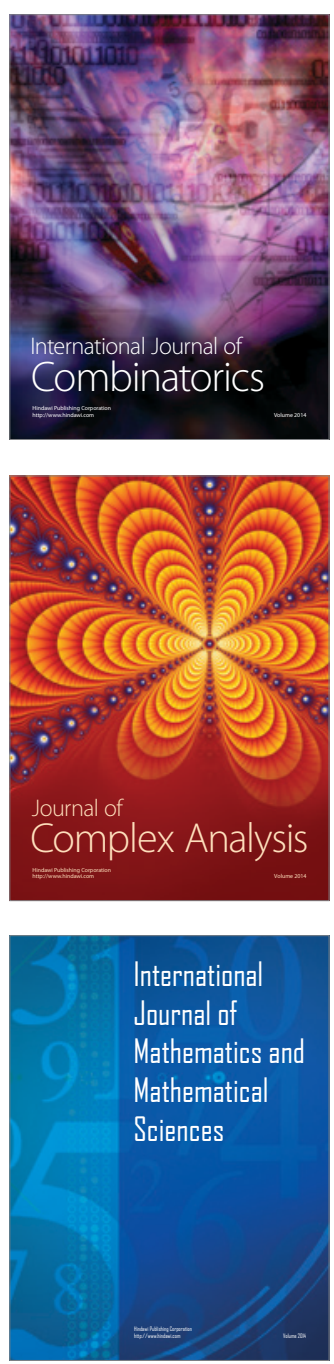
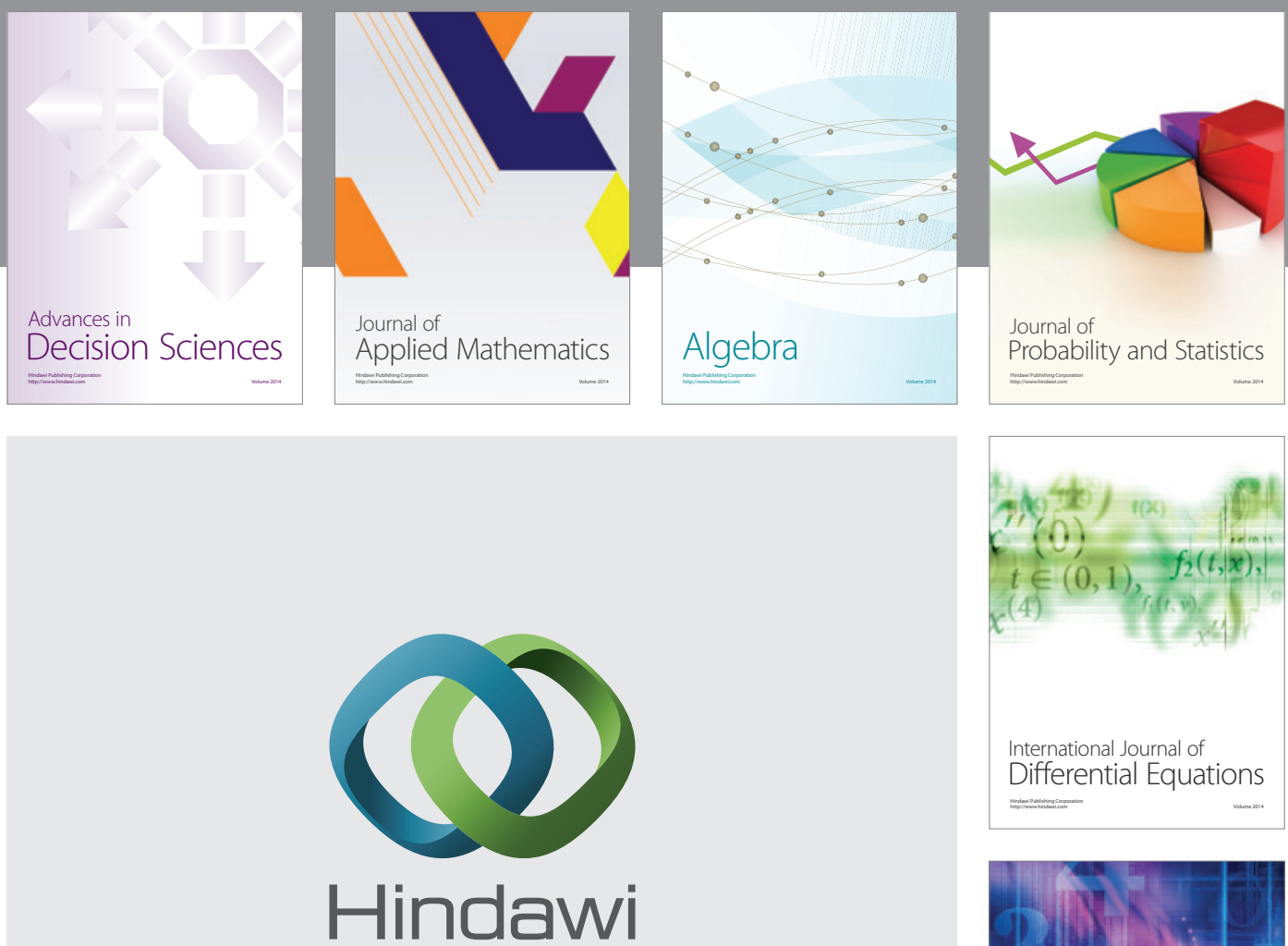

Submit your manuscripts at http://www.hindawi.com
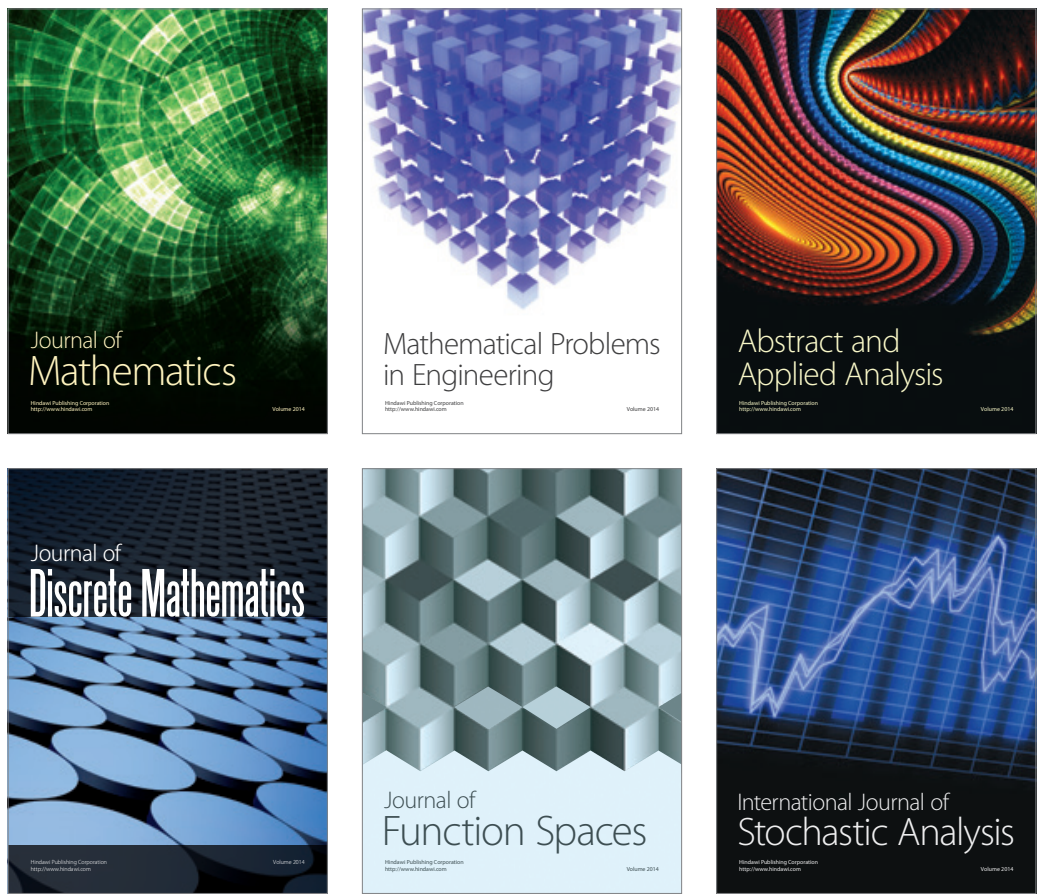

Journal of

Function Spaces

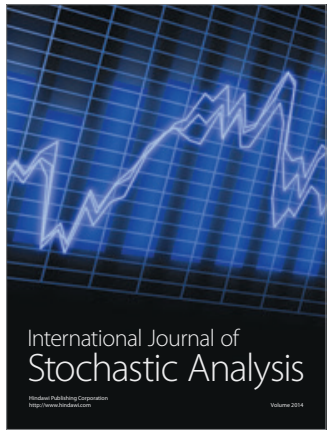

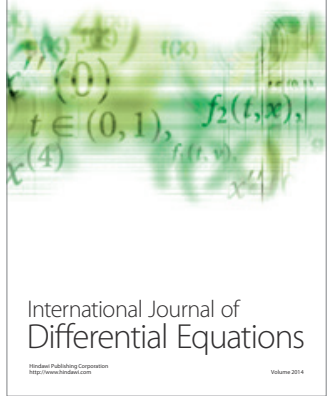
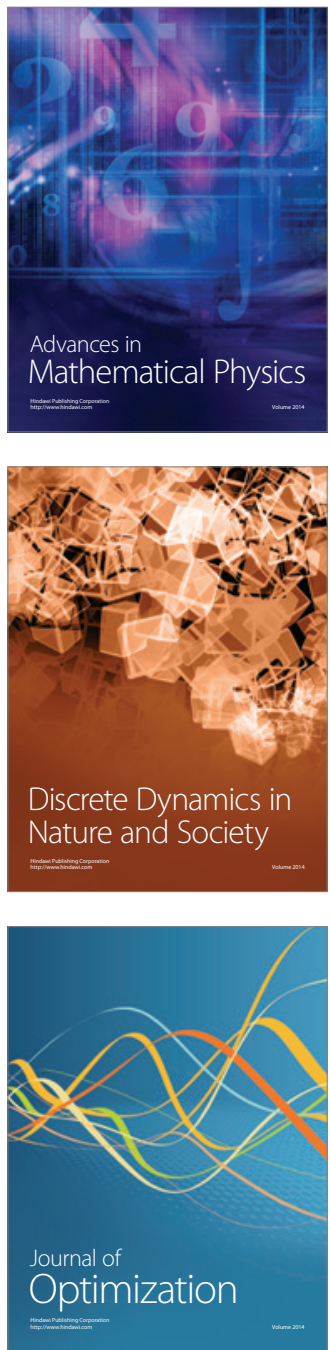